\title{
Drugs used in palliative medicine
}

\section{Introduction}

Palliative treatment is subject to slightly different rules of pharmacotherapy than chronic treatment. It must, though, be remembered that the first rule that guides the determination of treatment is always a proper and extensive interview and analysis of the patient's records. Some of the medicines described below are contraindicated in certain clinical conditions and diseases that may lead to a terminal condition. A good example is usually safe benzodiazepines, which due to their potential to relax skeletal muscles cannot be used in patients with myasthenia. ${ }^{1}$ Despite the slightly different rules of pharmacotherapy, the safety rules remain unchanged. Undoubtedly, the potential and long-term consequences of the treatment should be approached differently, but it is important to avoid aggressive pharmacotherapy. Any changes in doses or medications should be slow and gradual, so as not to cause additional and unnecessary stress to the patient's body. In most cases, palliative treatment applies to people already burdened with surgery, chemotherapy, or simply their advanced age and the accompanying diseases. Regarding author's clinical experience, it is often the case that terminally ill patients have a significantly lowered seizure threshold, unstable glycemic level, high or low blood pressure. In fact, these may be the consequences of numerous other drugs, organic brain damage, and often metastatic tumors. This fact should also be taken into account when determining the standard of pharmacotherapy. The use of individual pharmacological agents is, however, primarily determined by the clinical condition of the patient, the simultaneous administration of other pharmaceuticals, or simply the clinical sense and experience of the physician. There is no one or rigidly specified procedure. There are situations when patients are simply unable to receive the medicine orally, in which case the main criterion becomes a form that allows intravenous or intramuscular administration, and not the most appropriate clinical profile. It should be remembered that among medicines used in psychiatry not always a form other than oral is obvious.

\section{Aim and methods}

In this review article, the author will try to bring the issues regarding the use of drugs commonly used in psychiatry to treat the terminally ill patients. Authors have searched for literature in generally available databases. In this summary we have taken into account the knowledge contained in full-text publications in English or Polish. We reviewed particular aspects of the treatment used in palliative medicine with several drug groups. For simplicity, symptoms that might be reduced using different drugs have been grouped together. The description of each drug group will be made only once, although it happens to use several activities of one drug. A good example once again may be benzodiazepines. Drugs in this group have anticonvulsant, anxiolytic, sedative, hypnotic and myorelaxant effects.

\section{Anxiety disorders}

Anxiolytic activity is displayed by numerous drugs not only used in psychiatry. The first drug with proven and described anti-anxiety action has been the long unused meprobamate. Until the appearance of proper and above all safe, anxiolytic drugs, alcohol, bromine salts, opium and herbs had been used for this purpose. The benzodiazepines
Volume 2 Issue 2 - 2018

\author{
Grzegorz Opielak,' Tomasz Zuzak² \\ 'Department of Human Anatomy, Medical University of Lublin, \\ Poland \\ ${ }^{2}$ Student Research Group at The Department of Human \\ Anatomy, Medical University of Lublin, Poland
}

Correspondence: Grzegorz Opielak, Department of Human Anatomy, Medical University of Lublin,

4 Jaczewskiego St., 20-090 Lublin, Poland, Tel +48 60I 296965 ,

Email opielak@gmail.com

Received: January 15, 2018 | Published: April 19, 2018

are considered to be the true and appropriate group of anxiolytic drugs, described in more detail below. Anxiolytic activity of other drug groups is sometimes used rather imprudently. A very good example here can be a non-selective $\beta$-blocker - propranolol. The drug significantly suppresses the feeling of anxiety, but one must always pay attention to the possibility of interacting with other drugs or drugs from the same group (selective and non-selective blockers). Especially in the treatment of terminally ill patients, one should remember about the fact that they receive many other medicaments, which makes propranolol less useful. The sudden drop in pressure and pulse increase the already felt discomfort of the patient.

Another drug commonly used as an anti-anxiety agent may be hydroxyzine - a chemically derived diphenylmethane derivative. In this case, the problem may sometimes be either a bad and unpredictable tolerance or a lack of clinical effect in relation to the dose used. The advantage is the possibility of intramuscular injection. It should be remembered that hydroxyzine has a clear antihistamine and bronchodilatory effect, which may be important when combined with other drugs. Generally with due care, the drug is quite safe to use.

Also a safe form of treating anxiety is the use of not very high doses of antiepileptic drugs for this purpose: preparations of valproic acid, gabapentin or pregabalin. In addition, this kind of treatment is supported by the situation when we are dealing with anxiety, as a symptom arising as a result of damage to the CNS (cancer metastases, stroke-induced damages, traumatic lesions, etc.). The sudden emergence of anxiety in such a situation is sometimes a consequence of discharges in the cortex of the brain. One should be very careful to use only carbamazepine, it is not a good choice for palliative care. As mentioned above, the conditions of palliative treatment most often require poly-therapy, and the action of carbamazepine is associated with a burden on the liver and sometimes a change in the metabolism of other drugs. In addition, the risk of hematopoietic system involvement remains significant. ${ }^{2}$ Valproic acid derivatives are considered safe, although they also have their limitations. Liver damage or porphyria, basically exclude the possibility of valproates' administration. Also one should pay attention to clotting time and overall peripheral blood parameters. ${ }^{3}$ In cases of terminal treatment, the main problem of valproates, i.e. hair loss, goes far into the background. Gabapentin and pregabalin are used to treat anxiety, but the main use here seems 
to be the treatment of epilepsy when anxiety becomes secondary to epilepsy. Patients already receiving any of the preparations, with the appearance of periodic and sudden anxiety attacks may receive a slightly higher dose, the result is most often the withdrawal of anxiety symptoms and the increased patient's comfort.

One should not forget about the benzodiazepines. This is a very broad group of drugs that will be widely described in this paragraph, although the use of benzodiazepine derivatives goes far beyond anxiolytic treatment. In case of palliative treatment, the main disadvantage of this group of drugs, i.e. the gradual development of addiction and tolerance, according to the author, goes to the background. Nevertheless, one should always remember the above complications. ${ }^{4}$ In addition, low toxicity and good bioavailability are, undoubtedly, an asset. However, one should remember about the good and prudent choice of short and long-term measures. There is one, very important rule, short action means quick effect and strong saturation of receptors, while long-acting drugs are easily accumulated. The clinical picture of the use of benzodiazepine drugs, especially of short duration of action, sometimes resembles the one of antidepressants. In fact, the elevation of the mood is visible as a result of the abolition, or significant reduction of the nagging feeling of fear, and not the real effect of raising the level of serotonin. One should not that in the elderly and with organic lesions within the brain, paradoxical reactions often occur. Instead of sedation and hypnotic effect, we get a significant degree of agitation. Always remember this risk and if you get these types of symptoms, go for a medication from another group, for example, neuroleptics. A good choice turns out to be haloperidol, in addition easily accessible also in the form of injection.

Tricyclic drugs sometimes turn out to be useful. Doxepin is the most anti-anxiety drug in this group. At the same time, it should be mentioned that drugs of this group are generally difficult to use in palliative treatment. In addition to inducing arrhythmias, fluctuations in blood pressure, the absolute contraindications include damage to parenchymal organs involved in the metabolism of tricyclic drugs - liver and kidneys. If the patient is dealing with hyperthyroidism, Addison's disease, hematopoietic disorders, even in the face of deep depression, we should not use tricyclic drugs. ${ }^{5}$ Despite many contraindications, this group should not be totally discredited, because in certain clinical situations, however, it may be used, for example, in resistance to other antidepressants. Antipsychotics also have anxiolytic activity, but at the same time present many limitations resulting from their remaining actions. Generally, antipsychotics are used in two ways. The most important will be the antipsychotic activity described below, and the next antiemetic effect and if the patient's clinical condition requires antiemetics, a small dose of haloperidol or levomepromazine may be considered. The main obstacle, as in the case of tricyclic drugs, is the cardiac impact, namely QT prolongation. ${ }^{6}$

\section{Sleep disorders}

As mentioned above, hypnotic effects may be obtained by administering benzodiazepine drugs. This is the simplest and most effective method of hypnotic action. However, you should remember about the detailed verification of the other patient's orders. Benzodiazepines are safe, but it might be unpredictable to administer them with other drugs, especially those that have a suppressive effect on the respiratory center. The principle of treatment is simple - if patients have problems falling asleep, we can reach for short-acting forms (alprazolam, lorazepam), if the problem is the length and quality of sleep rather long-acting (diazepam, clorazepam). In the studies conducted among American doctors employed in hospices, it was shown that benzodiazepines are still very popular in the treatment of insomnia among terminally ill patients. The authors of this study underlined the broad spectrum of benzodiazepines. $65 \%$ of American doctors in the study said that benzodiazepines are helpful in chronic pain, insomnia and dying. ${ }^{7}$ Good hypnotic effects are provided by the use of some antidepressants. One of them is an old and proven trazodone, which does not have to be administered in large doses. Titleserine also works similarly. Of course, in case of treatment with opiate drugs, you can reach for tricyclic drugs, additionally enhancing the effect of the opiates. Doxepine, additionally acting clearly against anxiolytics, brings good results.

\section{Appetite loss}

In this case, it is mainly sulpiride that is considered as a medicine that simultaneously raises the mood and stimulates the appetite at the same time. Of course, it is difficult to recognize that as every case of appetite disorder is not the same. If it results from somatic reasons, the possibilities of impact are limited, if from the low mood of the patient, the proper use of antidepressants will also cause withdrawal of the disorder or lack of appetite, which in this case is secondary. In general, antihistamine drugs in the long run cause weight gain. ${ }^{8}$

\section{Pain disorders}

The essential treatment of pain is carried out in the terminal treatment most often with the use of opioid drugs and is subject to restrictions specific to this group of drugs. Good results, however, can be obtained through the potentiation of treatment and the association of opioids with tricyclic antidepressants. Positive results are also obtained using mianserin, a four-ring drug. The potential for analgesic therapy is seen with duloxetine. ${ }^{9}$ In a similar way, we can use gabapentin, which is sometimes used to treat polyneuropathy. It is worth recalling that well-chosen drugs and achieving painkillers contribute significantly to improving the comfort of life and increasing the mood of the patient. The essential analgesic treatment therefore affects the mental state, but in a manner appropriate to opiates. One should remember about the problem of cachexia among oncological patients. It is well known that nutritional therapy should be taken and its modification in time depending on the patient's condition. Nutritional treatment still seems to be insufficient to treat cachectic anemia. More and more often, new methods of treating anemia in terminally ill patients are searched. Deep hopes are associated with treatment based on erythropoietin, which has an effect on delaying the worsening of haematological parameters and weight loss in palliative patients. ${ }^{10}$

\section{Mood disorders}

Treatment of mood disorders is carried out mainly using antidepressants, above all - through the application of the tricyclics. As already mentioned, they are a perfect complement to opiate treatment, and you can also use mianserin, also safely associated with painkillers. With severe sleep disorders, trazodone can be given. Mirtazapine, a safe drug, is also worth considering, but it is important to remember quite often the symptoms of rapid sedation. ${ }^{11}$ In the absence of the use of antidepressants, an increase in the level of mood can be obtained by the use of neuroleptics. The use of atypical drugs, most often olanzapine or quetiapine, prove to be useful. On the other hand, haloperidol, sometimes used as a sedative agent, 
may have a depressing effect. The efficacy of valproic acid (VPA) and levetiracetam has also been demonstrated in palliative patients exhibiting moodiness. The good effects of these drugs appear to be independent of the primary cause of their administration in terminally ill patients. ${ }^{12}$

\section{Psychotic disorders and delirium}

In this case, it is difficult to talk about other drugs than antipsychotics. Of course, you may additionally add drugs that increase sedation, but in the case of psychotic disorders, essential treatment always takes place using neuroleptics. ${ }^{13}$ There is basically no rule about the most recommended means, this is done in relation to the current clinical situation of the patient. Rather, a strong neuroleptic should be used (eg, a Rossperidone $0.5-5 \mathrm{mg} / \mathrm{d}$, olanzapine $5-20 \mathrm{mg} /$ d), therefore the administered doses will be lower than in the case of weak neuroleptics (quetiapine $50-250 \mathrm{mg} / \mathrm{d}$ ). There are also no clear guidelines regarding the use of classical neuroleptics. A good example may have been the long-used and proven haloperidol. It is a neuroleptic with a strong antipsychotic effect. The main disadvantage may be extrapyramidal disorders. On the other hand, it slightly lowers the seizure threshold, which in case of patients burdened with other somatic diseases is of great importance. From other classical neuroleptics, chlorpromazine and promazine are used. Risperidone often induces hyperprolactinemia, and given the nature of palliative care and the high level of stress in patients, prolactin is still elevated. Olanzapine is a fairly safe neuroleptic, but it lowers seizure threshold and significantly disturbs lipid metabolism. It has been proven that in the treatment of delirium, olanzapine has the same effect as haloperidol - but this is still to be confirmed in further studies. ${ }^{14}$ In addition, it should be taken into account that in the case of the abovedescribed disorders, patients will not be willing to take medication, due to the lack of insight into the essence of the problem. Thus, it becomes easier to administer the drug in the form of a parenteral, and at least in Europe it is easier for such forms among classical neuroleptics.

\section{Conclusion}

At the end, one is obliged to always remember that regardless of the number of tips and schedules, the doctor always makes the final decision about the shape of pharmacotherapy. Of course, after conducting a thorough analysis of the clinical condition, the interview and the results of the patient's laboratory tests. The own experience of a physician dealing with terminally ill patients plays a key role. The ability of choosing the right therapy is still a big challenge. There is still a lack of research and new publications on treatment methods and algorithms for dealing with palliative patients. In terms of a holistic approach to the patient's problem, one cannot forget about the psychological aspect, which is very important for the terminally ill patients.

\section{Acknowledgements}

None.

\section{Conflict of interest}

The author declares no conflict of interest.

\section{References}

1. Haroutiunian S, Lecht S, Zur A, et al. The challenge of pain management in patients with myastenia gravis. J Pain Palliat Care Pharmacoter. 2009;23(3):242-260.

2. Pellock JM. Carbamazepine side effects in children and adults. Epilepsia 1987;28(Suppl 3):64-70.

3. Ruuskanen I, Kilpelainen HO, Riekkinen PJ. Side effects of sodium valproate during long-term treatment in epilepsy. Acta Neurol Scand. 1979;60(2):125-128.

4. Hirst A, Sloan R. Benzodiazepines and related drugs for insomnia in palliative care. Cochrane Database Syst Rev. 2002;(4).

5. Bilikiewicz A, Strzyżewski W. Psychiatria. Poland: PZWL Warszawa; 1992.

6. http://www.palliativecareguidelines.scot.nhs.uk/

7. Kamell A, Smith LK. Attitudes toward use of benzodiazepines among u.s. hospice clinicians: survey and review of the literature. J Palliat Med. 2016;19(5):516-522.

8. Kukreti P, Ali W, Jiloha RC. Rising trend of use of antidepressants induced non-puerperal lactation: a case report. J Clin Diagn Res. 2016;10(6).

9. Nübling G, Allmendinger S, Lorenzl S. Drug therapy of anxiety and fear in palliative care patients with cancer or other illnesses. Schmerz. 2012;26(5):537-541.

10. Penna F, Busquets S, Toledo M, et al. Erythropoietin administration partially prevents adipose tissue loss in experimental cancer cachexia models. J Lipid Res. 2013;54(11):3045-3051.

11.Pinder M. Farmakologiczne podstawy stosowania leków przeciwdepresyjnych w praktyce klinicznej. Farmakoterapia w psychiatrii i neurologii. 2000;1:58-73.

12. Thekdi SM, Trinidad A, Roth A. Psychopharmacology in Cancer. Curr Psychiatry Rep. 2015;17(1):529.

13. Bush SH, Tierney S, Lawlor PG. Clinical Assessment and Management of Delirium in the Palliative Care Setting. Drugs. 2017; 77(15):1623-1643.

14. Prommer E. Olanzapine: Palliative Medicine Update. Am J Hosp Palliat Med. 2013;30(1):75-82. 